\title{
Anterior Resection of the Rectum
}





\section{Single Surgical Procedures}

\section{A Colour Atlas of \\ Anterior Resection \\ of the Rectum}

Sir Hugh Lockhart-Mummery · Richard J. Heald · Ralph T. Hutchings

$$
\begin{array}{|l|}
\hline \mathrm{W} \\
\mathrm{DE} \\
\mathrm{G}
\end{array}
$$

Wolter de Gruyter · Berlin · New York 1984 
Sir Hugh Lockhart-Mummery KCVO, MD, MChir, FRCS; Consulting Surgeon, St. Thomas's Hospital, St. Mark's Hospital, London

Richard J.Heald MA, MChir, FRCS; Surgeon, Basingstoke Hospital,

Hampshire

Ralph T. Hutchings, Photographer; Formerly Chief Medical Laboratory,

Scientific Officer, Royal College of Surgeons of England

Copyright (C) Sir Hugh Lockhart-Mummery · Richard J. Heald .

Ralph T. Hutchings 1983

Original Publishers: Wolfe Medical Publications Ltd., $\cdot$ London

Exclusive co-publishers for the Federal Republic of Germany and Austria:

Walter de Gruyter \& Co., Genthiner Strasse 13, D-1000 Berlin 30. 1984.

Printed by Royal Smeets Offset b.v., Weert, Netherlands

Cover design: Rudolf Hübler

General Editor, Wolfe Surgical Atlases:

William F. Walker, DSc, ChM, FRCS (Eng.), FRCS (Edin.), FRS (Edin.)

CIP-Kurztitelaufnahme der Deutschen Bibliothek

Lockhart-Mummery, Hugh:

A colour atlas of anterior resection of the rectum /

Hugh Lockhart-Mummery; Richard J.Heald; Ralph T. Hutchings. -

Berlin; New York: de Gruyter, 1984.

(Single surgical procedures; 6 )

ISBN 3-11-010017-7

ISBN 3-11-010016-9 (Subskr.-Pr.)

NE: Heald, Richard J.; Hutchings, Raiph, T.:; GT

All rights reserved. The contents of this book, both photographic and textual, may not be reproduced in any form by print, photoprint, phototransparency, microfilm, microfiche or an other means, nor may it be included in any computer retrieval system, without written permission of the publisher.

Die Wiedergabe von Gebrauchsnamen, Warenbezejchnungen und deryleichen in diesem Buch berechtigt nicht zu der Annahme, daß solche Namen ohne weiteres von jedermann benutzt werden dürfen. Vielmehr handelt es sich häufig um gesetzlich geschützte.

eingetragene Warenzeichen, auch wenn sie nicht eigens als solche gekennzeichnet sind. 